\title{
Design of PI Controller with Lowpass Filter for Resonant Mechanical System
}

\author{
Member Guoguang Zhang (Osaka University) \\ Non-member Junji Furusho (Osaka University)
}

Key words: Extended Parameter Plane Method, PI Control, Lowpass Filter, Resonant Mechanical System.

\section{Introduction}

Motor drive systems driving a load through a shaft or a transmission system are widely found in industrial applications. Suppression of mechanical resonance is one of important topics in control of two-inertia system. It is shown that PI control is not able to provide a sufficient damping when the inertia ratio of load to motor becomes small ${ }^{(1)}$. One compensation method is to change the inertia ratio via the derivative feedback of motor speed (1) (2). In general, the derivative computing brings noise easily. In order to avoid the noise problem, PI controller combined with a lowpass filter is considered $^{(3)(4)(5)}$.

In practice, a simple and easy understanding design is desired. The extended parameter plane method proposed by authors ${ }^{(6)}$, provides an analytical design for control systems with three adjustable parameters. This paper presents how to use the method to analysis of speed control system with PI control plus a lowpass filter.

\section{Speed Control System}

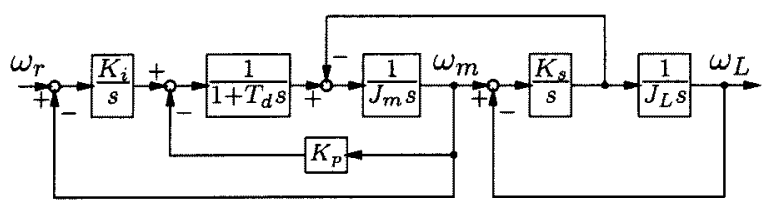

Fig. 1. Speed control system with PI controller plus a lowpass filter

A speed control system of two-inertia system, which employs PI control plus a one-order lowpass filter, is shown in Fig.1. $K_{p}$ and $K_{i}$ are proportional and integral feedback coefficients, respectively. $T_{d}$ is the time constant of the filter. In this figure,

$$
\begin{aligned}
& J_{m}: \text { Motor inertia, } \quad J_{L}: \text { Load inertia } \\
& \omega_{m}: \text { Motor speed, } \omega_{L}: \text { Load speed } \\
& K_{s}: \text { Torsional stiffness of drive shaft } \\
& \omega_{r}: \text { Reference input of motor speed }
\end{aligned}
$$

The transfer function from the reference input to the load speed is given by

$$
\frac{\omega_{L}(s)}{\omega_{r}(s)}=\frac{K_{i} \omega_{a}^{2}}{a_{5} s^{5}+a_{4} s^{4}+a_{3} s^{3}+a_{2} s^{2}+a_{1} s+a_{0}}
$$

where

$$
\begin{aligned}
& a_{5}=T_{d} \\
& a_{4}=1 \\
& a_{3}=\omega_{0}^{2} T_{d}+K_{p} / J_{m} \\
& a_{2}=\omega_{0}^{2}+K_{i} / J_{m} \\
& a_{1}=\omega_{a}^{2} K_{p} / J_{m} \\
& a_{0}=\omega_{a}^{2} K_{i} / J_{m}
\end{aligned}
$$

$\omega_{a}$ is antiresonant frequency, $\omega_{0}$ is resonant frequency, and $R$ is inertia ratio. They are defined as follows :

$$
\omega_{a}=\sqrt{\frac{K_{s}}{J_{L}}}, \quad \omega_{0}=\omega_{a} \sqrt{1+R}, \quad R=\frac{J_{L}}{J_{m}}
$$

The system in Eq.(1) is a fifth-order one, but there are only three adjustable coefficients $\left(K_{p}, K_{i}, T_{d}\right)$. It is not easy to design the system due to the less adjustable parameters.

\section{System Analysis}

The coefficients $a_{k}(k=0,1, \cdots, 5)$ in Eq.(1) are the linear functions of three parameters $K_{p}, K_{i}$ and $T_{d}$. Furthermore, $K_{p} / J_{m}, K_{i} / J_{m}$ and $T_{d}$ are considered as three designed parameters in analysis since $J_{m}$ is a constant. The extended parameter plane technique determines three adjustable parameter by assigning three poles in the complex plane ${ }^{(6)}$. 
The pair of complex poles $(\bullet)$ and the negative real pole $(+)$ are simply arranged to have an identical radius, and the poles $(\bullet)$ are fixed with the damping coefficient $=0.8$. For three different inertia ratios $R=0.75,0.5$ or 0.25 , the pole locations are shown in Fig.2 where the radius is varied from $0.5 \omega_{a}$ to $1.0 \omega_{a}$. It is seen that the locations of the designed poles obviously effect the pair of vibratory poles $(x)$. That is, as the three designed poles $(\bullet$ and + ) move away from the imaginary axis, the damping of the vibratory poles $(x)$ gets weak. It is also noted that the system shows a slight vibratory behavior as the inertia ratio is lowered.

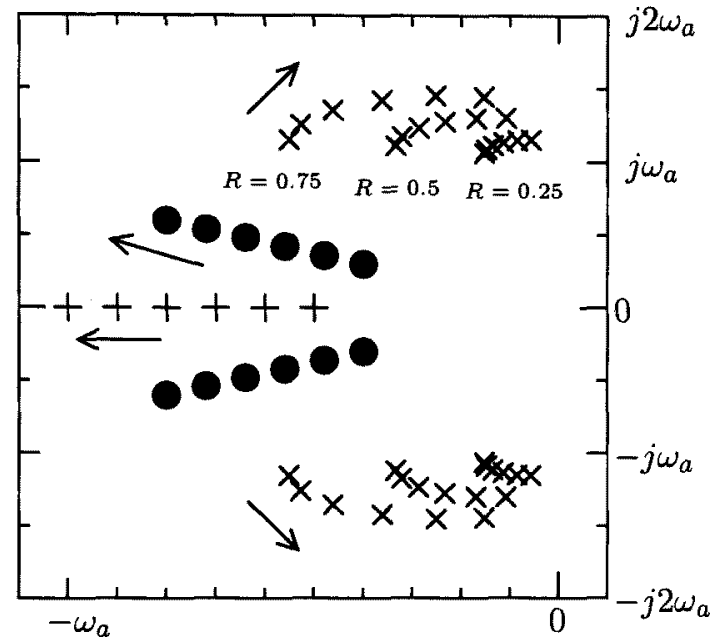

Fig. 2. Pole locations for $R=0.75,0.5,0.25$

The step responses are given in Fig.3, where the radius of the designed poles is selected as $0.7 \omega_{a}, 0.8 \omega_{a}$ or $0.9 \omega_{a}$, and $\omega_{a}$ is normalized as $1 \mathrm{rad} / \mathrm{s}$. The system shows good response for $R=0.75$ or 0.5 , compared with the one for $R=0.25$.

\section{Conclusions}

Based on the extended parameter plane method, the speed control system with PI controller plus a lowpass filter was analyzed. The lowpass filter clearly improves the response of the system when the inertia ratio is greater than 0.5 , however its effect gradually decreases as the inertia ratio gets small.

(Manuscript received April 23, 1999)

\section{References}

(1) G.Zhang and J.Furusho, "Systernatic Analysis and Design on Two-Inertia System", Trans. SICE Japan, Vol.35, No.1, pp.105-112, 1999

(2) G.Zhang and J.Furusho, "A. Practical PID Controller Design for Speed Servo System", Trans. IEE Japan, Vol.118-D, No.9, pp. 1095-1096, 1998

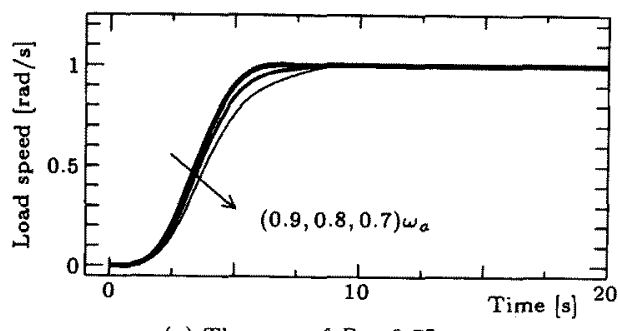

(a) The case of $R=0.75$

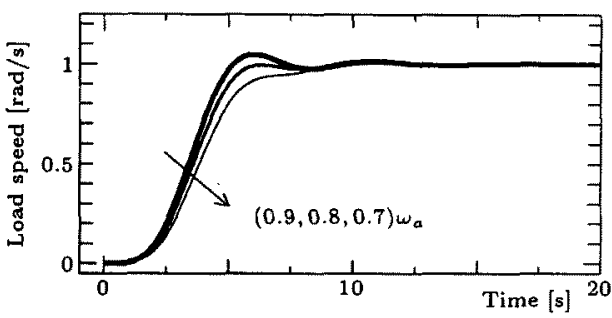

(b) The case of $R=0.5$

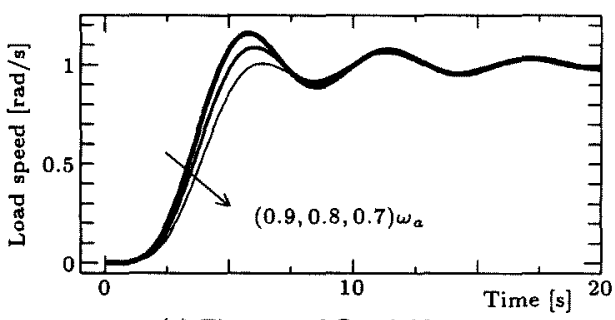

(c) The case of $R=0.25$

Fig. 3. Step responses for $R=0.75,0.5,0.25$

(3) S.Morimoto and Y.Takeda, "Vibration Suppression of Twomass System with First Order Lag Element", Trans. IEE Japan, Vol.114-D, No.9, pp.929-930,1994 (in Japanese)

(4) S.Morimoto and Y.Takeda, "Two-Degrees-of-Freedom Speed Control System of Resonant Mechanical System Based on $H_{\infty}$ Control Theory", Trans. IEE Japan, Vol.116-D, No.1, pp.6570,1996 (in Japanese)

(5) Y.Matsui and T.Takeuchi, "Design of Second-Order Controller for Resonant Mechanical System", Trans. IEE Japan, Vol.118-D, No.9, pp.1000-1007,1998 (in Japanese)

(6) G.Zhang and J.Furusho, "PID Controller Design for Flexible Joint Robots", Proc. of JSME D \& D '99 6th Symposium on Motion and Vibration Control, pp.284-289, March 1999, Chiba, Japan

Dr.Guoguang Zhang is a research associate of Osaka University. His research interests are control system design and its industrial applications, in particular, to electromechanical systems, motor drive systems and industrial robots.

Dr.Junji Furusho is a professor of the Department of Computer-Controlled Mechanical Systems at Osaka University. His research interests include robotics, motion control of mechanical systems and force display devices using intelligent fluids. 